\title{
Correction to: Population Dynamics of Nocturnal Lemurs in Littoral Forest Fragments: The Importance of Long-Term Monitoring
}

Sam Hyde Roberts ${ }^{1,2}$ - Retsiraiky J. Rossizela ${ }^{1} \cdot$ Tsimijaly H. Longosoa $^{1}$. Kathryn Strang ${ }^{1} \cdot$ Lucia Chmurova $^{1}$ - Vincent Nijman ${ }^{2}$. Giuseppe Donati ${ }^{2}$

Published online: 3 January 2022

(c) The Author(s) 2022

\section{Correction to: International Journal of Primatology \\ https://doi.org/10.1007/s10764-021-00243-1}

The original version of this article unfortunately contained mistakes in Figure 2, Table III and Table IV. The legends in Figure 2 panel a were missing and the entries in Table III and Table IV were inadvertently mis-aligned with the species.

The original article can be found online at https://doi.org/10.1007/s10764-021-00243-1.

\section{Sam Hyde Roberts}

samhyderoberts@gmail.com; sam.roberts@seedmadagascar.org

1 SEED Madagascar, London, UK

2 Oxford Brookes University, Oxford, UK 
The correct version of Figure 2, Table III and Table IV are presented below:

Figure 2
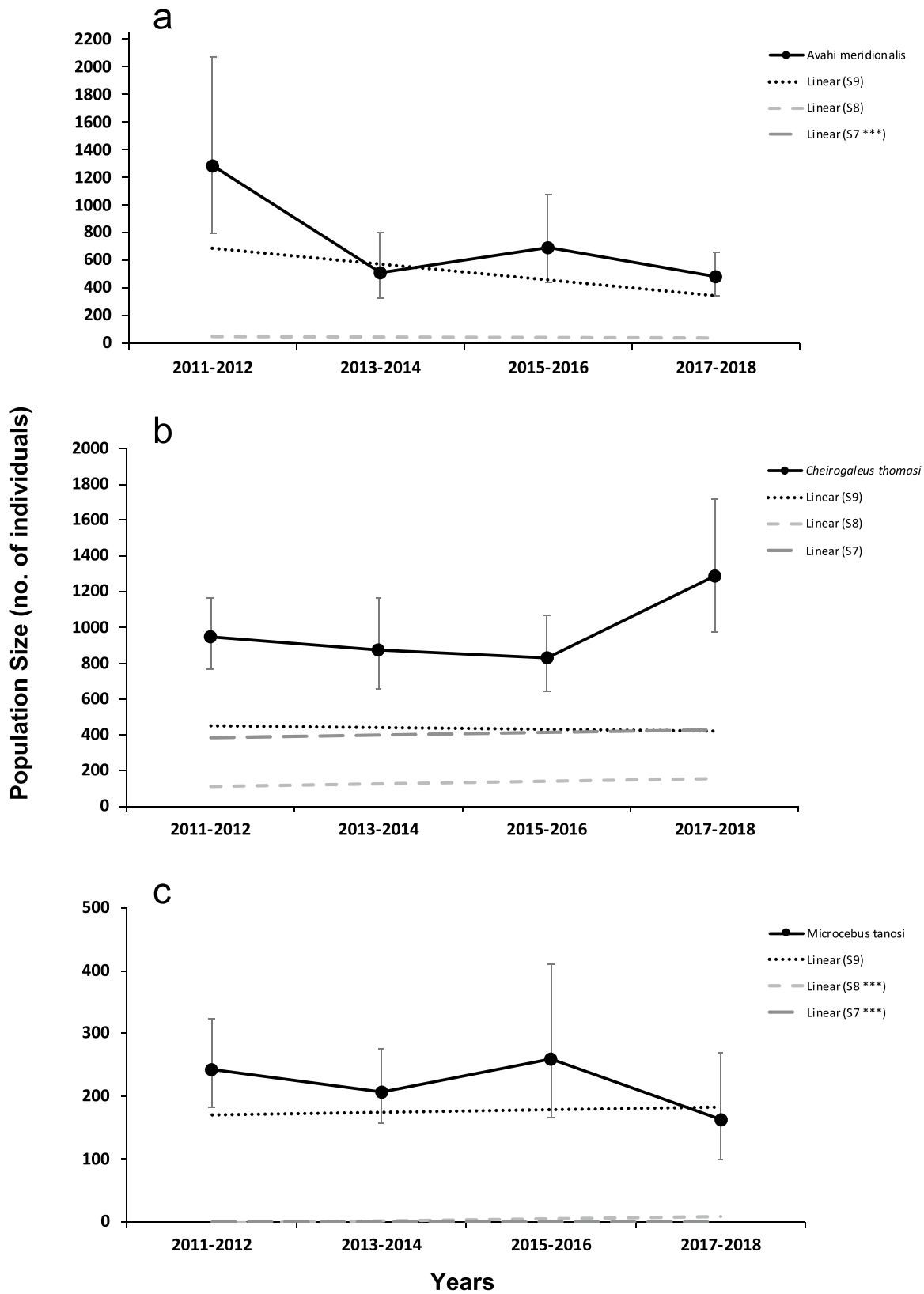
Table III Results of a type III test of GLMM fixed effects and interactions for three nocturnal lemur species in Sainte Luce, Madagascar, during an 8-yr study (2011-2018)

\begin{tabular}{|c|c|c|c|c|c|c|}
\hline & Fixed Effect & & $F$ & df & $\mathrm{P}$ & $n$ \\
\hline \multirow[t]{6}{*}{ Avahi meridionalis } & Year & & 0.195 & 3 & 0.900 & 483 \\
\hline & Fragment & & 69.804 & 2 & $<0.001$ & 483 \\
\hline & \multirow[t]{3}{*}{ Year*Fragment } & S9 & 2.270 & 3 & 0.080 & 483 \\
\hline & & S8 & 1.404 & 3 & 0.241 & 483 \\
\hline & & S7 & 0.929 & 3 & 0.427 & 483 \\
\hline & Effect of Protection & & 0.577 & 1 & 0.448 & 483 \\
\hline \multirow[t]{7}{*}{ Cheirogaleus thomasi } & Year & & 0.151 & 3 & 0.929 & 284 \\
\hline & Fragment & & 4.514 & 3 & 0.004 & 284 \\
\hline & \multirow[t]{4}{*}{ Year*Fragment } & S9 & 1.117 & 3 & 0.342 & 284 \\
\hline & & S8 N & 0.341 & 3 & 0.796 & 284 \\
\hline & & S8 S & 0.371 & 3 & 0.774 & 284 \\
\hline & & S7 & 0.367 & 3 & 0.777 & 284 \\
\hline & Effect of Protection & & 0.261 & 1 & 0.610 & 284 \\
\hline \multirow[t]{6}{*}{ Microcebus tanosi } & Year & & 0.562 & 3 & 0.640 & 483 \\
\hline & Fragment & & 2.512 & 2 & 0.082 & 483 \\
\hline & \multirow[t]{3}{*}{ Year*Fragment } & S9 & 0.767 & 3 & 0.513 & 483 \\
\hline & & S8 & 0.478 & 3 & 0.698 & 483 \\
\hline & & S7 & 0.429 & 3 & 0.732 & 483 \\
\hline & Effect of Protection & & 0.130 & 1 & 0.719 & 483 \\
\hline
\end{tabular}

*Represents interactions between effects. $n$ indicates number of transect repeats. Boldface numbers highlight significant results

Table IV Pairwise comparisons based on GLMM model estimates showing only significant results

\begin{tabular}{lllllll}
\hline & Fixed Effect & Pairwise comparison & $t$ & df & P & $n$ \\
\hline Avahi meridionalis & Fragment & S7 - S8 & -7.485 & 471 & $<0.001$ & 483 \\
& & S7 - S9 & -9.387 & 471 & $<0.001$ & 483 \\
& & S8 - S9 & -4.194 & 471 & 0.022 & 483 \\
& Year* Fragment (S9) & 2011/12-2013/14 & 2.552 & 471 & 0.011 & 483 \\
Cheirogaleus thomasi & Fragment & S7-S8S & 3.046 & 268 & 0.003 & 284 \\
& & S7 - S9 & 2.509 & 268 & 0.013 & 284 \\
& & S8N-S8S & 2.637 & 268 & 0.009 & 284 \\
Microcebus tanosi & Fragment & S8N-S9 & 2.028 & 268 & 0.044 & 284 \\
\hline
\end{tabular}

$t$ values indicate direction of result. $n$ indicates number of transect repeats

The original article has been corrected. 